\title{
The Emerging Role of Sestrin2 in Cell Metabolism, and Cardiovascular and Age-Related Diseases
}

\author{
Wanqing Sun ${ }^{1,2, \#, ~ Y i s h i ~ W a n g ~}{ }^{3, \#, ~ Y a n g ~ Z h e n g ', ~ N a n h u ~ Q u a n ~}{ }^{1, *}$ \\ ${ }^{1}$ Cardiovascular Center, First Affiliated Hospital of Jilin University, Changchun, Jilin, China \\ ${ }^{2}$ Fuwai Hospital, National Center of Cardiovascular Diseases, Beijing, China \\ ${ }^{3}$ Department of Physiology and Pathophysiology, Fourth Military Medical University, Xi'an, Shaanxi, China
}

[Received January 6, 2019; Revised March 20, 2019; Accepted March 24, 2019]

\begin{abstract}
Sestrins (Sesns), including Sesn1, Sesn2, and Sesn3, are cysteine sulfinyl reductases that play critical roles in the regulation of peroxide signaling and oxidant defense. Sesn 2 is thought to regulate cell growth, metabolism, and survival response to various stresses, and act as a positive regulator of autophagy. The antioxidative and anti-aging roles of Sesn2 have been the focus of many recent studies. The role of Sesn2 in cellular metabolism and cardiovascular and age-related diseases must be analyzed and discussed. In this review, we discuss the physiological and pathophysiological roles and signaling pathways of Sesn 2 in different stress-related conditions, such as oxidative stress, genotoxic stress, and hypoxia. Sesn2 is also involved in aging, cancer, diabetes, and ischemic heart disease. Understanding the actions of Sesn2 in cell metabolism and age-related diseases will provide new evidence for future experimental research and aid in the development of novel therapeutic strategies for Sesn2-related diseases.
\end{abstract}

Key words: sestrin2, cell metabolism, aging, cancer, myocardial ischemia

\section{Introduction}

Aging is an eternal theme in human life and many people seek a return to their youth, or at least a way of slowing the aging process. Aging is a complicated process, and many pathological changes are involved, including dysfunctional cell metabolism, the attenuation of host defenses, and memory loss [1-3] Consequently, aging can lead to various diseases, such as cancer, diabetes, and myopathy $[4,5]$. Although much progress has been made in anti-aging research, this mysterious process is not fully understood, and new therapeutic targets are urgently needed. Aging, once considered irreversible as a major risk factor for many chronic diseases, is in fact extremely malleable. Strategies to delay and potentially reverse the ageing process have long been a focus of research even if molecular mechanisms are not fully understood. As aging progresses, individuals are less tolerant of various stresses. Notably, stress-inducible proteins have been claimed as involved in aging.

Sestrins (Sesns) are a group of highly conserved stress-inducible proteins that respond to a variety of environmental stresses including oxidative stress, DNA damage, and hypoxia [6]. Two main biological functions of Sesns have been identified. First, Sesns act as antioxidants that can decrease the accumulation of reactive oxygen species (ROS) [7]. Second, they function as inhibitors of mammalian targets of rapamycin complex 1 (mTORC1) [7, 8]. Sesns bind to kelch-like ECHassociated protein 1 (Keap1) and p62/SQSTM, and suppress the autophagic degradation of Keap1, leading to nuclear factor (erythroid-derived 2)-like 2 (Nrf2)

*Correspondence should be addressed to: Dr. Nanhu Quan, Cardiovascular Center, First Affiliated Hospital of Jilin University, Changchun, Jilin 130000, China; E-mail: naohoo@hotmail.com. \#These authors contributed equally to this work.

Copyright: $\odot 2019$ Sun W et al. This is an open-access article distributed under the terms of the Creative Commons Attribution License, which permits unrestricted use, distribution, and reproduction in any medium, provided the original author and source are credited. 
activation [9]. Moreover, Sesns can suppress mTORC1 activity through the activation of AMPK [8, 10, 11]. Based on these functions, Sesns are thought to attenuate various age-related metabolic disorders, including insulin resistance, glucose intolerance, mitochondrial dysfunction, muscle degeneration, and cardiac dysfunction [8, 12-15]. Recent studies using Sesn2 and Sesn 3 knockout (KO) mice have confirmed the role of Sesns in the suppression of age- and obesity-associated metabolic diseases $[9,12]$.

In this review, we summarize the latest advances regarding the association of Sesns with cardiovascular and age-related metabolic diseases. First, we review the general background of Sesn2. Then, we discuss the role of Sesn2 in several pathophysiological conditions, such as oxidative stress, genotoxic stress, and hypoxia. In addition, we illustrate several signaling pathways that are associated with Sesn2. Finally, we highlight certain Sesn2-related conditions, including aging, cancer, diabetes, and ischemic heart disease. This review will provide a better understanding of the actions of Sesn 2 in cell metabolism and age-related diseases as well as new evidence for future experimental research and promote the role of Sesn2 as a novel therapeutic target.

\section{General background of Sesn2}

In 1999, Velasco-Miguel et al. first reported that PA26, known as Sesnl, was a novel target of the tumor suppressor $\mathrm{p} 53$ and has properties common to the growth arrest and DNA damage-inducible (GADD) family of stress-response genes, which suggests that Sesn1 acts as a potential novel regulator of cellular growth [16]. In 2002, Budanov et al. used cDNA microarray hybridization to identify novel genes that participate in cellular responses to prolonged hypoxia. They found a novel gene, Hi95 (Sesn2), which shares marked homology with p53regulated GADD family member PA26 [17]. Increased Hi95 transcription was observed in response to prolonged hypoxia, DNA damage, and oxidative stress, but not to hyperthermia or serum starvation. Furthermore, the induction of Hi95 by prolonged hypoxia or oxidative stress is most likely p53-independent and is induced by DNA damage (gamma- or ultraviolet [UV]-irradiation, or doxorubicin) in a p53-dependent manner. They then demonstrated that the over-expression of Hi95 full-length cDNA is toxic among many types of cultured cells and leads either to apoptotic death or sensitization to serum starvation and DNA damage. However, conditional overexpression of the Hi95 cDNA in MCF7-tet-off cells was found to be protective against cell death triggered by hypoxia/glucose deprivation or $\mathrm{H}_{2} \mathrm{O}_{2}$. Their study suggests that Sesn2 is involved in the regulation of cell survival in response to various types of stress. In addition,
Sesn2 has been suggested to be involved in the pathogenesis of heterotaxia, which is characterized by an abnormal left-right axis formation and reversed left-right polarity of one or more organ systems [18]. They found that, in a patient with heterotaxia, the PA26 gene is disrupted by the $6 \mathrm{q} 21$ breakpoint.

As a target of $p 53$, which is the guardian of the genome, Sesn 2 protein acts as an antioxidant, which is responsible for diminishing the accumulation of ROS [19] and inhibits mTORC1 signaling [8]. Both the accumulation of ROS and the activation of mTORC1 are strongly associated with aging and age-related diseases $[20,21]$, indicating that Sesn2 is a novel target for antiaging research. While accumulating evidence suggests an association between Sesn2 and aging, new reports have demonstrated that Sesn2 plays a role in cancer, inflammation, and neurological diseases [22-26]. In the current review, we discuss the regulation of Sesn2 and its role in the regulation of metabolism and aging.

\section{Regulation of Sesn2 expression under various conditions}

In response to various insults, cells respond by adjusting to changing conditions to survive. For example, autophagy will occur if a cell is starved; the cell will survive to a certain extent for some time with the help of autophagy. Moreover, a cell will undergo anoxic respiration in hypoxic conditions. Through these adjustments, a cell can save energy to enable its survival under harsh conditions. The expression of Sesn 2 is stressinducible and involved in the regulation of cell viability in response to different stress conditions [17]. Therefore, it is important to elucidate the regulatory mechanism of Sesn 2 expression under various types of stress.

\subsection{Oxidative stress}

Oxidative stress is characterized by the overproduction of ROS and reactive nitrogen species (RNS), which leads to dysfunction of the cell. Oxidative stress can not only oxidize DNA and RNA, but can also damage other molecules including proteins and lipids [20]. The accumulation of the products of oxidative stress causes aging [20], cell apoptosis [27], neurodegeneration [28], and metabolic syndrome [29]. The members of the Sesn family are induced by oxidative stress via different mechanisms. Sesn 1 is induced in a p53-dependent manner, while Sesn 2 can be induced by oxidative stress in a p53-dependent manner [30]. Recent studies have demonstrated that the induction of Sesn 2 by oxidative stress is dependent on Nrf2 and activator protein-1 [31, 32]. Sesn 2 activates Nrf2 by removing its inhibitor Keap1, which leads to activation of the antioxidant system. Sesn2 
is induced by NMDA receptor activation in neurons in a CCAAT-enhancer-binding protein beta (c/EBP $\beta)$ dependent manner [33]. The Cys125 residue of Sesn2, which is conserved throughout the Sesn-family, is critical for the antioxidant activity of Sesn2 [19]. Sesn2 is proven to inhibit uncoupling protein 1 expression by suppressing ROS-mediated activation of p38 mitogen-activated protein kinase in brown adipose tissue [34]. Additionally, Sesn 2 may decrease renal oxidative stress and mediate the dopamine D2 receptor-induced inhibition of ROS production [35]. Therefore, Sesn2 plays a key role in maintaining cellular homeostasis during oxidative stress.

\subsection{Genotoxic stress}

Genotoxic stress is suggested to boost aging, and interfere with DNA damage repair [36]. Genotoxic stress can be triggered by the overproduction of DNA-damaging molecules, such as ROS, RNS, reactive carbonyl species, lipid peroxidation products, and DNA-alkylating agents. Excessive genotoxic stress can activate DNA damagesensing signaling pathways, including activation of tumor suppressor p53, which confers cell cycle-inhibitory and pro-apoptotic activities to eliminate damaged cells. As a DNA damage-inducible protein, Sesn 2 play a critical role in the response to genotoxic stress [37]. Sesn2 is induced upon DNA damage (gamma- and UV-irradiation and genotoxic metabolites) through the activation of p53 [16, 17]. Budanov et al. demonstrated that Sesn 2 activates AMPK signaling and inhibits mTOR upon genotoxic challenge, while Sesn2-deficient mice fail to inhibit mTOR signaling [10]. The inhibition of mTOR signaling can minimize the synthesis of new proteins and membranes during genotoxic stress, which ultimately saves energy for DNA repair.

\subsection{Hypoxia}

Hypoxia, that is, insufficient oxygen availability, is another stimulus that can induce Sesn2 activation [17]. Sesn2 (Hi95) was first identified as a gene that was activated by hypoxia in human neuroblastoma cells [17]. Transcriptional activation of Sesn2 upon hypoxia is reported to be hypoxia-inducible factor-1-dependent in mouse epithelial tracheal cells [38]. A recent study indicated that Sesn2 activation is independent of p53 but requires the PI3K/Akt pathway in response to energetic stress induced by 2-deoxyglucose (2-DG) [39]. The inhibition of Akt, as well as loss of Sesn2, sensitizes cells to 2-DG-induced apoptosis. Moreover, the rescue of Sesn 2 partially reverses the pro-apoptotic effects of 2-DG. In addition, metformin (inhibitor of mitochondrial respiration), induces the expression of Sesn2 via an unknown mechanism [39].

\subsection{Endoplasmic reticulum stress}

Endoplasmic reticulum (ER) stress is characterized by an accumulation of misfolded proteins. Upon ER stress, the attenuation of protein translation is essential for maintaining tissue homeostasis. Park et al. demonstrated that Sesn2 expression is induced by the ER stressactivated transcription factor c/EBP $\beta$. Once induced, Sesn2 subsequently prevents protein synthesis by inhibiting mTORC1. Conversely, if Sesn2 is lacking, cells are highly susceptible to ER stress-associated cell death due to the continued translation of large amounts of protein [14]. A recent study indicated that the induction of Sesn2 under ER stress is dependent on activating transcription factor 6, and Sesn2 plays a hepatoprotective role in the presence of excess ER stress by inhibiting CCAAT-enhancer-binding protein homologous protein, c-Jun N-terminal kinase (JNK), and p38 [40]. ER stress is an aspect of the pathogenesis of aging and a variety of human diseases, such as cardiovascular diseases; however, the transcription factors involved in the induction of Sesn2 by ER stress and the protective mechanisms triggered by Sesn2 against ER stressmediated cell death require further exploration.

\section{Sesn 2 and signaling pathways}

\subsection{Keap1/Nrf2 signaling pathway}

Keap1 is a cysteine-rich protein that represses $\mathrm{Nrf} 2$ activity [41]. Under quiescent conditions, Nrf2 localizes in the cytoplasm and binds to Keap1 [42]. Once activated by oxidative stress, Nrf2 disassociates from Keap1, translocates to the nucleus, and binds to the antioxidant response element sequences in the promoter regions of a set of cytoprotective genes, such as hemoxygenase 1, NADPH:quinone oxidoreductase, and glutathione-S transferase, and activates their transcription [43]. In the nucleus, Nrf2 can also be degraded by the ubiquitinproteasome system. Nrf2 activation contributes to protection against a variety of diseases, including cardiovascular and neurodegenerative diseases [44-48]. Therefore, Keap1/Nrf2 signaling plays a critical role in maintaining homeostasis in cells under oxidative stress.

It has been suggested that Sesn2, activated by oxidative stress, increases the expression of sulfiredoxin through the activation of Nrf2 [9]. In addition, Sesn2 activates $\mathrm{Nrf} 2$ by promoting p62-dependent autophagic degradation of Keap1, thus leading to Nrf2 activation, and decreased susceptibility of the liver to oxidative damage [9]. Sesn2 decreases the accumulation of ROS and stimulates anti-oxidant defenses via Keap1/Nrf2 signaling activation. 


\subsection{AMPK/mTORC1 signaling pathway}

The target of rapamycin (TOR), an evolutionary conserved protein, is a pivotal regulator of cell growth, proliferation, metabolism, and autophagy [7]. TOR was first identified as a protein kinase inhibited by rapamycin [49-51]. Mammalian TOR (mTOR) is present in two distinct complexes, namely, mTORC1, which is sensitive to rapamycin, and mTORC2, which is not. mTORC1 consists of mTOR raptor, PRAS40, and mLST8, and is responsible for the regulation of cell growth and protein synthesis [52, 53]. mTORC2 consists of mTOR, Rictor, Sin1, Protor, and mLST8, and regulates the actin cytoskeleton and cell spreading [53-55]. The regulation of mTORC1 is dependent on tuberous sclerosis 1 and 2 proteins (TSC1 and TSC2), which form a stable heterodimer (TSC1:TSC2 complex), of which the TSC2 subunit acts as a GTPase-activating protein for the small GTPase Rheb, which negatively controls Rheb and activates mTOR [50, 56]. The activity of TSC2 is regulated by several protein kinases, such as Akt, extracellular signal-regulated kinase, ribosomal S6 kinase, and AMP-activated protein kinase (AMPK) [57, 58].

AMPK is an important nutrient-sensing protein kinase that plays a critical role in maintaining metabolic homeostasis [59, 60]. Nutrient starvation, caloric restriction, and exercise can lead to ATP depletion and an increase in the AMP to ATP ratio, which triggers the phosphorylation of TSC2 [61,62]. Other research suggests that AMPK can directly inhibit mTORC1 activity through the phosphorylation of its regulatory subunit Raptor [63]. The mechanisms underlying AMPK activation include phosphorylation by its upstream kinases such as liver kinase B1 (LKB1), $\mathrm{Ca}^{2+} /$ calmodulin-dependent kinase, and transforming growth factor- $\beta$-activated kinase 1 , and through interaction with regulatory proteins such as kinase suppressor of Ras $2[64,65]$.

When induced by certain stresses, Sesn2 inhibits mTORC1 through the activation of AMPK [10]. Studies have shown that Sesn-deficient cells and tissues exhibit lower AMPK and higher mTORC1 activities under both normal and stress conditions [8, 10, 66]. Although it remains unclear how Sesn2 activates AMPK, Sanli et al. suggested that Sesn2 facilitates the LKB1-dependent phosphorylation of AMPK and functions as a guanine nucleotide dissociation inhibitor for Rag GTPases to control mTORC1 signaling $[67,68]$. Sesn 2 promotes the metabolic adaptation of cells in response to various insults and mediates genotoxic stress-induced activation of AMPK and the suppression of mTORC1 [10]. Furthermore, as a target gene of p53, Sesn2 acts as a critical link between genotoxic stress, p53, and the mTORC1 signaling pathway. Sesn2 activation inhibits
mTORC1 activity, leading to inhibited phosphorylation of S6K and eukaryotic translation initiation factor 4Ebinding protein (4E-BP) [10]. By inhibiting the phosphorylation of 4E-BP, Sesn2 enhances its interactions with eIF-4E and inhibits the expression of growth regulatory proteins, such as cyclin D1 and c-Myc [69-71]. More importantly, mTORC1 inhibits autophagy, which plays a critical role in the regulation of cell viability by providing necessary nutrients during starvation and monitoring the health and integrity of organelles, especially mitochondria. Dysfunctional autophagy leads to the accumulation of damaged mitochondria, which produces ROS and thereby triggers oxidative stress and cellular damage [72]. mTORC1 inhibits autophagy through the phosphorylation of ATG1 (ULK1) protein, and ATG13 protein [73]. As an inhibitor of mTORC1, Sesn 2 promotes autophagy. The cessation of protein synthesis and the promotion of autophagy are both significant events for the suppression of cell growth during genotoxic stress, and the saved energy can be used for DNA repair, thereby boosting the survival of cells in response to stress insults. Therefore, AMPK/mTORC1 signaling pathway is critical for the role of Sesn2 in controlling cell metabolism and survival under conditions of stress.

More intriguingly, Lee et al. demonstrated that persistent TORC1 activation in Drosophila induces $d S e s n$ through ROS-dependent activation of JNK and FoxO [8]. Furthermore, TORC1 generates ROS to induce $d S e s n$; this is prevented by feeding animals vitamin $\mathrm{E}$, an antioxidant. Here, we can see that Sesn 2 induced by ROS can scavenge ROS accumulation, suggesting the important role of the TORC1-Sesn2-AMPK-TORC1 feedback loop in maintaining cell metabolism and homeostasis.

\section{Sesn2 and aging}

The hallmarks of aging include the accumulation of aberrant proteins and oxidative stress, dysfunction of cellular metabolism and organs, and defective homeostasis. Moreover, aging is associated with many diseases such as diabetes, cardiovascular diseases, cancer, and neurodegenerative diseases, such as Alzheimer's disease and Parkinson's disease. It has been shown that the activation of AMPK, suppression of mTORC1, and stimulation of autophagic signaling are beneficial for extending both lifespan and healthspan [74-78]. Therefore, since Sesn2 can activate AMPK, suppress mTORC1, and stimulate autophagy, it could also slow the aging process.

Lee et al. demonstrated that a loss of Sesn in Drosophila leads to age-associated pathologies including triglyceride accumulation, mitochondrial dysfunction, muscle degeneration, and cardiac malfunction, all of 
which could be reversed by pharmacological activation of AMPK or inhibition of TOR [8]. dSesn-null adults have a higher level of triglycerides, which were decreased after ectopic expression of $d S e s n$. In addition, $d S e s n$-null adults show decreased AMPK and increased TOR activities. After feeding $d S e s n$-null adults with AMPK-activators such as AICAR (5-aminoimidazole-4-carboxamide 1- $\beta$ D-ribofuranoside) or metformin, or the TOR-inhibitor rapamycin, reduced accumulation of triglycerides was found $[79,80]$. In addition, the heart function of $d S e s n-$ null flies is compromised, as demonstrated by arrhythmia and decreased heart rate prevented by feeding AICAR or rapamycin to the flies, which indicated that they had low AMPK activity or high TORC1 activity [8]. $d S e s n$-null flies also undergo age-associated muscle degeneration. Twenty-day-old $d S e s n$-null flies show degeneration of thoracic muscles with loss of sarcomeric structure, and diffused sarcomere boundaries, which can only be found in old wild-type flies ( $\sim 90$ days). Apart from these signs, muscle cells from 5-day-old $d S e s n$-null flies have rounded, enlarged, or disorganized mitochondria, resulting in ROS accumulation, which can be prevented by feeding the flies vitamin E. Importantly, feeding dSesn-null flies with AMPK activators and rapamycin also prevents muscle degeneration, suggesting that AMPK/TOR signaling is critical for the prevention of mitochondrial dysfunction and the maintenance of muscular homeostasis during aging. By activating AMPK and inhibiting TORC1, Sesns can reprogram cells to attenuate stress injury by reducing anabolism and enhancing autophagy. Sesns can act as antioxidants to inhibit oxidative damage, promote autophagy, eliminate mitochondrial dysfunction, or inhibit mitochondrial metabolism, which produces ROS. Therefore, Sesns can be thought of as physiological controllers that can attenuate accelerated stress-dependent aging [81]. More importantly, it is worth noting that Sesns are also expressed under normal unstressed conditions, even in the absence of any environmental stress, the $d S$ Sesn knockout mutants show an accelerated aging phenotype [81]. Therefore, Sesns may provide baseline protection to reduce the damage caused by physiological damage, which is an inevitable consequence of basic life processes, such as oxidative respiration and DNA replication.

\section{Sesn2 and diabetes}

As an evolutionarily conserved and nutrient-sensing protein kinase, mTORC1 plays a pivotal role in the regulation of cell metabolism. Chronic mTORC1 activation is induced by nutritional abundance, resulting in enhanced protein and lipid synthesis and inhibited autophagy $[82,83]$. By activating S6K, mTORC1 can contribute to insulin resistance by inhibiting the phosphorylation of insulin receptor substrates, and attenuating the PI3K/Akt signaling pathway induced by insulin [84]. On the other hand, liver-specific S6K depletion protects against hepatic steatosis and systemic insulin resistance [84]. Recent studies have suggested that mTORC1 also phosphorylates and activates growth factor receptor-bound protein 10 , which has been identified as an inhibitor of PI3K/Akt signaling pathway $[85,86]$. Defective autophagy, which can be caused by mTORC1 activation, promotes ER stress and results in insulin resistance [87]. In addition, ER stress leads to the suppression of insulin receptor signaling through hyperactivation of JNK [88]. Therefore, the prolonged activation of mTORC1 contributes to insulin resistance and type II diabetes.

Sesn2, as the inhibitor of mTORC1, is considered to improve insulin resistance. A recent report suggests that exercise induces AMPK and Sesn2 interaction, and leads to increased insulin-sensitivity through autophagy [89]. Sesn2 deficiency enhances obesity-induced insulin resistance and the progression of diabetes. Additionally, Sesn $2^{-/}$mice display defective insulin responsiveness and compromised insulin-stimulated PI3K-AKT signaling in the liver [12]. Notably, Sesn2 maintains insulin sensitivity by promoting AMPK activation in the liver, and loss of Sesn 2 aggravates hepatosteatosis caused by obesity [12]. Therefore, the regulation of Sesn2 activity may provide an alternative approach for the prevention of insulin resistance, obesity, and diabetes.

\section{Sesn2 and cancer}

Cancer is strongly associated with oxidative stress, metabolic dysregulation, and gene mutation. Cancer cells need to synthesize new proteins and lipids to produce new cells [90]. It has been reported that TORC1 signaling pathway is often activated in human cancers and rapamycin exhibits anti-tumor activity through the inhibition of TORC1 [52]. A recent study suggests that mTORC1 upregulates Golgi protein 73, which promotes the proliferation and migration of hepatocellular carcinoma cells [91]. The mTORC1 inhibitor everolimus confers antitumor activity in vitro, and produces tumor responses in patients with relapsed T-cell lymphoma [92]. Using rapamycin, translation elongation is inhibited, which limits intestinal tumor initiation and growth [93].

Because Sesn2 can suppress TORC1 activation, Sesn2 may confer tumor suppressor activity. Decreased expression of Sesn 2 predicts an unfavorable prognosis in colorectal cancer [94]. Sesn2 is shown to be an important regulator of mTORC1 signaling and can inhibit colon carcinogenesis [22]. Sesn2-deficient mouse embryonic fibroblasts were significantly more susceptible to oncogenic transformation than wild type counterparts, 
suggesting that Sesn2 may have tumor suppressive activity [10]. Sesn2 inhibition leads to the acceleration of A549 lung carcinoma cell growth [30]. These findings indicate that Sesn 2 confers tumor a suppressive function. However, Sesn 2 is also important for maintaining the viability of cancers under several conditions. As a ROS scavenger, Sesn 2 can be activated in cancer cells to attenuate oxidative stress. Moreover, the activation of Sesn2 in tumor cells can activate autophagy, which facilitates tumor cell growth under conditions of limited nutrients and oxygen [95]. Sesn2 positively regulates Akt signaling and survival in human squamous cell carcinoma (SCC) and melanoma cells in response to UVB stress and chemotherapeutics, suggesting that Sesn2 may promote tumorigenesis and chemoresistance in SCC and melanoma [96]. The advantageous or disadvantageous roles of Sesn2 merit further investigation. The results might lead to a novel approach for radiotherapy or chemotherapy in cancers through the regulation of Sesn2 activity.

\section{Sesn2 and ischemic heart disease}

Myocardial ischemia occurs when the heart is subjected to hypoxia. Myocardial ischemia and reperfusion cause bursts of ROS production, leading to cardiac arrhythmia and heart failure [97]. In addition, autophagy is impaired in cardiac ischemia-reperfusion injury and the restoration of autophagosome clearance attenuates reoxygenation- induced cell death [98]. In the Drosophila heart, loss of dSesn function leads to cardiac arrhythmia [8]. Sesn2 also promotes LKB1-mediated AMPK activation in the ischemic heart [13]. By activating autophagy, Sesn2 may confer cardioprotection against ischemia. Since autophagy has a key role in ischemic preconditioning against ischemia [99-102], Sesn2 might confer cardioprotection via this route. Therefore, Sesn 2 is a novel therapeutic target to attenuate ROS accumulation and enhance autophagy in response to heart ischemia.

As is widely accepted, aging is accompanied by intensive susceptibility of the myocardium to ischemia/reperfusion injury [103]. This susceptibility of the aged heart is closely related to impaired autophagy and decreased activity of AMPK [104]. Our recent study found that the expression of Sesn 2 decreased with aging, and this led to reduced ischemic AMPK activation, significantly impaired downstream glucose uptake, and the oxidation rate. The binding affinity between Sesn 2 and AMPK upstream LKB1 is also impaired in aged hearts during ischemia [105]. We found that Sestrin2 prevents age-related intolerance to ischemia and reperfusion injury by modulating substrate metabolism. These findings further confirm that Sesn2 is a potential target that could prevent age-related susceptibility to ischemic heart disease $[105,106]$.

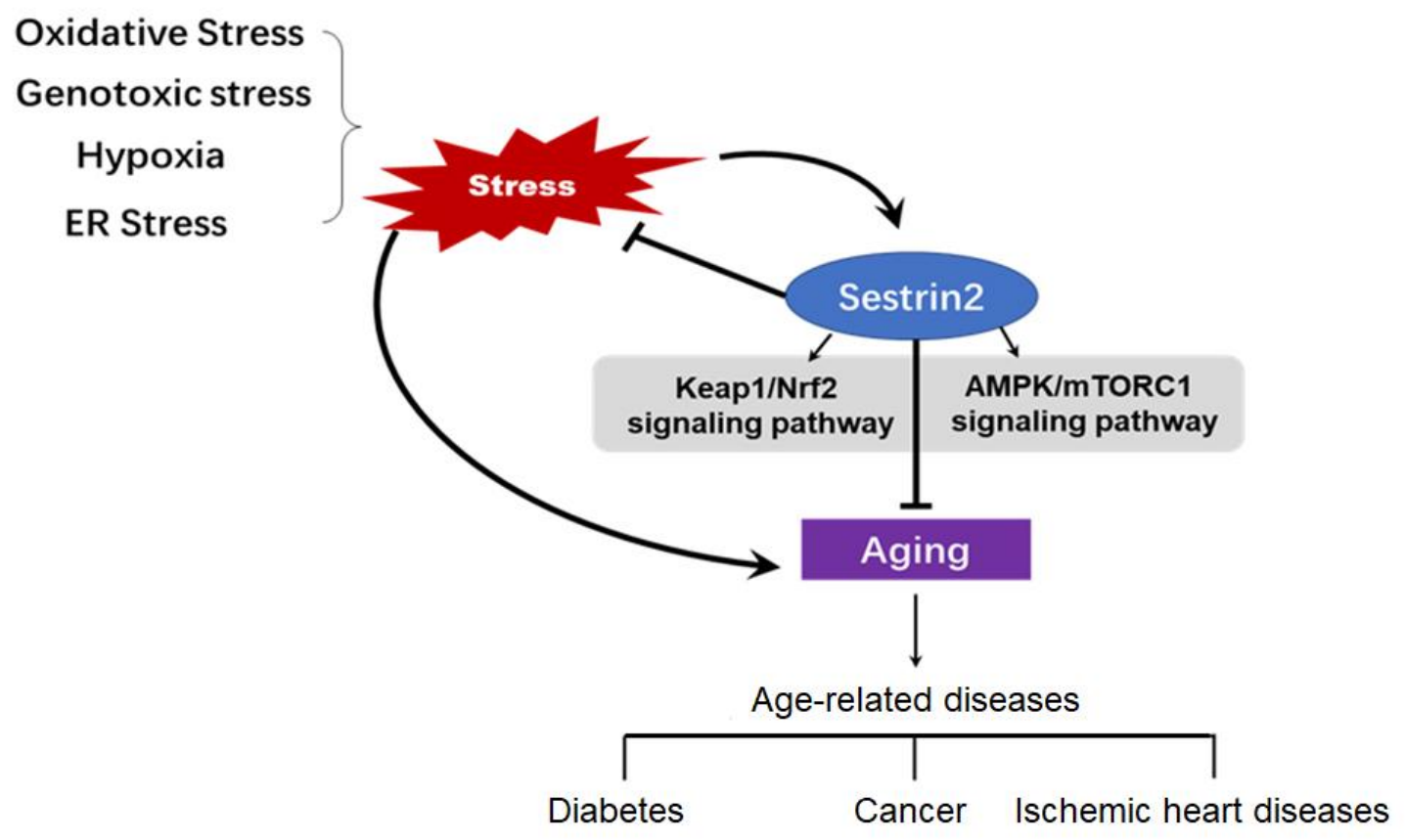

Figure 1. Summary of the merging role of sestrin2 in cell metabolism, cardiovascular and aging-reaged diseases 


\section{Perspectives}

Data obtained from animal models indicate that Sesn2 is a promising target for the treatment of cardiovascular diseases in human patients. In this research, we briefly reviewed how Sesn2 may act as a suppressor of aging effects associated with responsive to cell metabolism, stressful stimuli, and age-related cardiovascular diseases (Figure 1). As a target of p53, Sesn2 is considered to be an important regulator of metabolism and aging. Sesn2 can diminish ROS accumulation, and activate autophagy, thus suppressing age-related diseases, obesity, diabetes, neurodegenerative diseases, and cancer. The ability to evaluate whether Sesn 2 is activated or inhibited in a given condition and to clarify its functional significance needs to be improved. Sesn2 provides a novel therapeutic target for the prevention of age-related diseases and metabolic disorders. Further research is needed to throw light upon the specific underlying mechanisms by which the functions of Sesn2 are achieved.

\section{Acknowledgments}

This work was supported by the National Natural Science Foundation of China (Grant No. 81870289 to Dr. Quan, and Grant No. 81500264 to Dr. Sun). We thank Ji Li, University of Mississippi Medical Center, for the helpful support.

\section{Competing Interests}

The authors declare that no competing interests exist.

\section{References}

[1] Sun N, Youle RJ, Finkel T (2016). The Mitochondrial Basis of Aging. Mol Cell, 61:654-666.

[2] Gkikas I, Petratou D, Tavernarakis N (2014). Longevity pathways and memory aging. Front Genet, 5:155.

[3] Cuervo AM, Macian F (2014). Autophagy and the immune function in aging. Curr Opin Immunol, 29:97104.

[4] Carruba G, Cocciadiferro L, Di Cristina A, Granata OM, Dolcemascolo C, Campisi I, et al. (2016). Nutrition, aging and cancer: lessons from dietary intervention studies. Immun Ageing, 13:13.

[5] Blokh D, Stambler I (2017). The application of information theory for the research of aging and agingrelated diseases. Prog Neurobiol, 157:158-173.

[6] Lee JH, Budanov AV, Karin M (2013). Sestrins orchestrate cellular metabolism to attenuate aging. Cell Metab, 18:792-801.

[7] Budanov AV, Lee JH, Karin M (2010). Stressin' Sestrins take an aging fight. EMBO Mol Med, 2:388-
400.

[8] Lee JH, Budanov AV, Park EJ, Birse R, Kim TE, Perkins GA, et al. (2010). Sestrin as a feedback inhibitor of TOR that prevents age-related pathologies. Science, 327:1223-1228.

[9] Bae SH, Sung SH, Oh SY, Lim JM, Lee SK, Park YN, et al. (2013). Sestrins activate Nrf2 by promoting p62dependent autophagic degradation of Keap1 and prevent oxidative liver damage. Cell Metab, 17:73-84.

[10] Budanov AV, Karin M (2008). p53 target genes sestrin1 and sestrin2 connect genotoxic stress and mTOR signaling. Cell, 134:451-460.

[11] Chen CC, Jeon SM, Bhaskar PT, Nogueira V, Sundararajan D, Tonic I, et al. (2010). FoxOs inhibit mTORC1 and activate Akt by inducing the expression of Sestrin3 and Rictor. Dev Cell, 18:592-604.

[12] Lee JH, Budanov AV, Talukdar S, Park EJ, Park HL, Park HW, et al. (2012). Maintenance of metabolic homeostasis by Sestrin2 and Sestrin3. Cell Metab, 16:311-321.

[13] Morrison A, Chen L, Wang J, Zhang M, Yang H, Ma $\mathrm{Y}$, et al. (2015). Sestrin2 promotes LKB1-mediated AMPK activation in the ischemic heart. FASEB J, 29:408-417.

[14] Park HW, Park H, Ro SH, Jang I, Semple IA, Kim DN, et al. (2014). Hepatoprotective role of Sestrin2 against chronic ER stress. Nat Commun, 5:4233.

[15] Tao R, Xiong X, Liangpunsakul S, Dong XC (2015). Sestrin 3 protein enhances hepatic insulin sensitivity by direct activation of the mTORC2-Akt signaling. Diabetes, 64:1211-1223.

[16] Velasco-Miguel S, Buckbinder L, Jean P, Gelbert L, Talbott R, Laidlaw J, et al. (1999). PA26, a novel target of the 553 tumor suppressor and member of the GADD family of DNA damage and growth arrest inducible genes. Oncogene, 18:127-137.

[17] Budanov AV, Shoshani T, Faerman A, Zelin E, Kamer I, Kalinski H, et al. (2002). Identification of a novel stress-responsive gene $\mathrm{Hi95}$ involved in regulation of cell viability. Oncogene, 21:6017-6031.

[18] Peeters H, Debeer P, Bairoch A, Wilquet V, Huysmans C, Parthoens E, et al. (2003). PA26 is a candidate gene for heterotaxia in humans: identification of a novel PA26-related gene family in human and mouse. Hum. Genet., 112:573-580.

[19] Budanov AV, Sablina AA, Feinstein E, Koonin EV, Chumakov PM (2004). Regeneration of peroxiredoxins by p53-regulated sestrins, homologs of bacterial AhpD. Science, 304:596-600.

[20] Finkel T, Holbrook NJ (2000). Oxidants, oxidative stress and the biology of ageing. Nature, 408:239-247. Stanfel MN, Shamieh LS, Kaeberlein M, Kennedy BK (2009). The TOR pathway comes of age. Biochim Biophys Acta, 1790:1067-1074.

[22] Ro SH, Xue X, Ramakrishnan SK, Cho CS, Namkoong S, Jang I, et al. (2016). Tumor suppressive role of sestrin2 during colitis and colon carcinogenesis. Elife, 5:e12204.

[23] Yang JH, Kim KM, Kim MG, Seo KH, Han JY, Ka SO, et al. (2015). Role of sestrin2 in the regulation of 
proinflammatory signaling in macrophages. Free Radic Biol Med, 78:156-167.

[24] Wu CL, Chen SD, Yin JH, Hwang CS, Yang DI (2016). Nuclear Factor-kappaB-Dependent Sestrin2 Induction Mediates the Antioxidant Effects of BDNF Against Mitochondrial Inhibition in Rat Cortical Neurons. Mol Neurobiol, 53:4126-4142.

[25] Chuang YC, Yang JL, Yang DI, Lin TK, Liou CW, Chen SD (2015). Roles of Sestrin2 and Ribosomal Protein S6 in Transient Global Ischemia-Induced Hippocampal Neuronal Injury. Int J Mol Sci, 16: 26406-26416.

[26] Kim GT, Lee SH, Kim YM (2013). Quercetin Regulates Sestrin 2-AMPK-mTOR Signaling Pathway and Induces Apoptosis via Increased Intracellular ROS in HCT116 Colon Cancer Cells. J Cancer Prev, 18:264-270.

[27] Cao Y, Gao Y, Xu S, Bao J, Lin Y, Luo X, et al. (2016). Glutamate carboxypeptidase II gene knockout attenuates oxidative stress and cortical apoptosis after traumatic brain injury. BMC Neurosci, 17:15.

[28] Emerit J, Edeas M, Bricaire F (2004). Neurodegenerative diseases and oxidative stress. Biomed Pharmacother, 58:39-46.

[29] Roberts CK, Sindhu KK (2009). Oxidative stress and metabolic syndrome. Life Sci, 84:705-712.

[30] Sablina AA, Budanov AV, Ilyinskaya GV, Agapova LS, Kravchenko JE, Chumakov PM (2005). The antioxidant function of the p53 tumor suppressor. Nat Med, 11:1306-1313.

[31] Shin BY, Jin SH, Cho IJ, Ki SH (2012). Nrf2-ARE pathway regulates induction of Sestrin-2 expression. Free Radic Biol Med, 53:834-841.

[32] Zhang XY, Wu XQ, Deng R, Sun T, Feng GK, Zhu XF (2013). Upregulation of sestrin 2 expression via JNK pathway activation contributes to autophagy induction in cancer cells. Cell Signal, 25:150-158.

[33] Papadia S, Soriano FX, Leveille F, Martel MA, Dakin $\mathrm{KA}$, Hansen HH, et al. (2008). Synaptic NMDA receptor activity boosts intrinsic antioxidant defenses. Nat Neurosci, 11:476-487.

[34] Ro S, Nam M, Jang I, Park H, Park H, Semple I, et al. (2014). Sestrin2 inhibits uncoupling protein 1 expression through suppressing reactive oxygen species. Proc. Natl. Acad. Sci. U.S.A., 111:7849-7854.

[35] Yang Y, Cuevas S, Yang S, Villar VA, Escano C, Asico $\mathrm{L}$, et al. (2014). Sestrin2 decreases renal oxidative stress, lowers blood pressure, and mediates dopamine D2 receptor-induced inhibition of reactive oxygen species production. Hypertension, 64:825-832.

[36] Hoeijmakers JH (2009). DNA damage, aging, and cancer. N Engl J Med, 361:1475-1485.

[37] Hay N (2008). p53 strikes mTORC1 by employing sestrins. Cell Metab, 8:184-185.

[38] Olson N, Hristova M, Heintz NH, Lounsbury KM, van der Vliet A (2011). Activation of hypoxia-inducible factor-1 protects airway epithelium against oxidantinduced barrier dysfunction. Am J Physiol Lung Cell Mol Physiol, 301:L993-L1002.

[39] Ben-Sahra I, Dirat B, Laurent K, Puissant A, Auberger
P, Budanov A, et al. (2013). Sestrin2 integrates Akt and mTOR signaling to protect cells against energetic stress-induced death. Cell Death Differ, 20:611-619.

[40] Jegal KH, Park SM, Cho SS, Byun SH, Ku SK, Kim SC, et al. (2017). Activating transcription factor 6dependent sestrin 2 induction ameliorates ER stressmediated liver injury. Biochim Biophys Acta, 1864:1295-1307.

[41] Bae SH, Woo HA, Sung SH, Lee HE, Lee SK, Kil IS, et al. (2009). Induction of sulfiredoxin via an Nrf2dependent pathway and hyperoxidation of peroxiredoxin III in the lungs of mice exposed to hyperoxia. Antioxid Redox Signal, 11:937-948.

[42] Kaspar JW, Niture SK, Jaiswal AK (2009). Nrf2:INrf2 (Keap1) signaling in oxidative stress. Free Radic Biol Med, 47:1304-1309.

[43] Kensler TW, Wakabayashi N, Biswal S (2007). Cell survival responses to environmental stresses via the Keap1-Nrf2-ARE pathway. Annu Rev Pharmacol Toxicol, 47:89-116.

[44] Ashrafian H, Czibik G, Bellahcene M, Aksentijević D, Smith A, Mitchell S, et al. (2012). Fumarate is cardioprotective via activation of the Nrf2 antioxidant pathway. Cell Metab., 15:361-371.

[45] Hybertson BM, Gao B, Bose SK, McCord JM (2011). Oxidative stress in health and disease: the therapeutic potential of Nrf2 activation. Mol Aspects Med, 32:234-246.

[46] Piantadosi CA, Carraway MS, Babiker A, Suliman HB (2008). Heme oxygenase-1 regulates cardiac mitochondrial biogenesis via Nrf2-mediated transcriptional control of nuclear respiratory factor-1. Circ Res, 103:1232-1240.

[47] Fan W, Tang Z, Chen D, Moughon D, Ding X, Chen S, et al. (2010). Keap1 facilitates p62-mediated ubiquitin aggregate clearance via autophagy. Autophagy, 6:614621.

[48] Buendia I, Michalska P, Navarro E, Gameiro I, Egea J, Leon R (2016). Nrf2-ARE pathway: An emerging target against oxidative stress and neuroinflammation in neurodegenerative diseases. Pharmacol Ther, 157:84-104.

[49] Alexander A, Cai SL, Kim J, Nanez A, Sahin M, MacLean KH, et al. (2010). ATM signals to TSC2 in the cytoplasm to regulate $\mathrm{mTORC1}$ in response to ROS. Proc Natl Acad Sci U S A, 107:4153-4158.

[50] Wullschleger S, Loewith R, Hall MN (2006). TOR signaling in growth and metabolism. Cell, 124:471484.

[51] Blagosklonny MV (2008). Aging: ROS or TOR. Cell Cycle, 7:3344-3354.

[52] Guertin DA, Sabatini DM (2007). Defining the role of mTOR in cancer. Cancer Cell, 12:9-22.

[53] Yang Q, Guan KL (2007). Expanding mTOR signaling. Cell Res, 17:666-681.

[54] Astrinidis A, Cash TP, Hunter DS, Walker CL, Chernoff J, Henske EP (2002). Tuberin, the tuberous sclerosis complex 2 tumor suppressor gene product, regulates Rho activation, cell adhesion and migration. Oncogene, 21:8470-8476. 
[55] Sarbassov D, Ali S, Kim D, Guertin D, Latek R, Erdjument-Bromage H, et al. (2004). Rictor, a novel binding partner of mTOR, defines a rapamycininsensitive and raptor-independent pathway that regulates the cytoskeleton. Curr. Biol., 14:1296-1302.

[56] Laplante M, Sabatini D (2009). mTOR signaling at a glance. J. Cell. Sci., 122:3589-3594.

[57] Corradetti M, Guan K (2006). Upstream of the mammalian target of rapamycin: do all roads pass through mTOR? Oncogene, 25:6347-6360.

[58] Kwiatkowski D, Manning B (2005). Tuberous sclerosis: a GAP at the crossroads of multiple signaling pathways. Hum. Mol. Genet., 14 Spec No. 2:R251-258.

[59] Kishton R, Barnes C, Nichols A, Cohen S, Gerriets V, Siska P, et al. (2016). AMPK Is Essential to Balance Glycolysis and Mitochondrial Metabolism to Control T-ALL Cell Stress and Survival. Cell Metab., 23:649662.

[60] Toyama EQ, Herzig S, Courchet J, Lewis TL, Jr., Loson OC, Hellberg K, et al. (2016). Metabolism. AMP-activated protein kinase mediates mitochondrial fission in response to energy stress. Science, 351:275281.

[61] Shaw RJ (2009). LKB1 and AMP-activated protein kinase control of mTOR signalling and growth. Acta Physiol (Oxf), 196:65-80.

[62] Inoki K, Zhu T, Guan KL (2003). TSC2 mediates cellular energy response to control cell growth and survival. Cell, 115:577-590.

[63] Gwinn DM, Shackelford DB, Egan DF, Mihaylova MM, Mery A, Vasquez DS, et al. (2008). AMPK phosphorylation of raptor mediates a metabolic checkpoint. Mol Cell, 30:214-226.

[64] Costanzo-Garvey D, Pfluger P, Dougherty M, Stock J, Boehm M, Chaika O, et al. (2009). KSR2 is an essential regulator of AMP kinase, energy expenditure, and insulin sensitivity. Cell Metab., 10:366-378.

[65] Wang W, Guan KL (2009). AMP-activated protein kinase and cancer. Acta Physiol (Oxf), 196:55-63.

[66] Wempe F, De-Zolt S, Koli K, Bangsow T, Parajuli N, Dumitrascu R, et al. (2010). Inactivation of sestrin 2 induces TGF-beta signaling and partially rescues pulmonary emphysema in a mouse model of COPD. Dis Model Mech, 3:246-253.

[67] Sanli T, Linher-Melville K, Tsakiridis T, Singh G (2012). Sestrin 2 modulates AMPK subunit expression and its response to ionizing radiation in breast cancer cells. PLoS One, 7:e32035.

[68] Peng M, Yin N, Li M (2014). Sestrins function as guanine nucleotide dissociation inhibitors for $\mathrm{Rag}$ GTPases to control mTORC1 signaling. Cell, 159:122-133.

[69] Averous J, Fonseca BD, Proud CG (2008). Regulation of cyclin D1 expression by mTORC1 signaling requires eukaryotic initiation factor 4E-binding protein 1. Oncogene, 27:1106-1113.

[70] Averous J, Proud C (2006). When translation meets transformation: the mTOR story. Oncogene, 25:64236435 .
[71] Mamane Y, Petroulakis E, Rong L, Yoshida K, Ler L, Sonenberg N (2004). eIF4E--from translation to transformation. Oncogene, 23:3172-3179.

[72] Gottlieb R, Carreira R (2010). Autophagy in health and disease. 5. Mitophagy as a way of life. Am. J. Physiol., Cell Physiol., 299:C203-210.

[73] Hosokawa N, Hara T, Kaizuka T, Kishi C, Takamura A, Miura Y, et al. (2009). Nutrient-dependent mTORC1 association with the ULK1-Atg13-FIP200 complex required for autophagy. Mol Biol Cell, 20:1981-1991.

[74] Mair W, Morantte I, Rodrigues A, Manning G, Montminy M, Shaw R, et al. (2011). Lifespan extension induced by AMPK and calcineurin is mediated by CRTC-1 and CREB. Nature, 470:404-408 Hofmann JW, Zhao X, De Cecco M, Peterson AL, Pagliaroli L, Manivannan J, et al. (2015). Reduced expression of MYC increases longevity and enhances healthspan. Cell, 160:477-488.

[76] Wen X, Klionsky D (2016). Autophagy is a key factor in maintaining the regenerative capacity of muscle stem cells by promoting quiescence and preventing senescence. Autophagy, 12:617-618.

[77] Yamamoto T, Takabatake Y, Kimura T, Takahashi A, Namba T, Matsuda J, et al. (2016). Time-dependent dysregulation of autophagy: Implications in aging and mitochondrial homeostasis in the kidney proximal tubule. Autophagy, 12:801-813.

[78] Seah N, de Magalhaes Filho C, Petrashen A, Henderson H, Laguer J, Gonzalez J, et al. (2016). Autophagy-mediated longevity is modulated by lipoprotein biogenesis. Autophagy, 12:261-272.

[79] Hay N, Sonenberg N (2004). Upstream and downstream of mTOR. Genes Dev, 18:1926-1945.

[80] Towler M, Hardie D (2007). AMP-activated protein kinase in metabolic control and insulin signaling. Circ. Res., 100:328-341.

[81] Lee JH, Bodmer R, Bier E, Karin M (2010). Sestrins at the crossroad between stress and aging. Aging (Albany NY), 2:369-374.

[82] Um SH, D'Alessio D, Thomas G (2006). Nutrient overload, insulin resistance, and ribosomal protein $\mathrm{S} 6$ kinase 1, S6K1. Cell Metab, 3:393-402.

[83] Zoncu R, Efeyan A, Sabatini D (2011). mTOR: from growth signal integration to cancer, diabetes and ageing. Nat. Rev. Mol. Cell Biol., 12:21-35.

[84] Bae E, Xu J, Oh D, Bandyopadhyay G, Lagakos W, Keshwani M, et al. (2012). Liver-specific p70 S6 kinase depletion protects against hepatic steatosis and systemic insulin resistance. J. Biol. Chem., 287:18769-18780.

[85] Hsu PP, Kang SA, Rameseder J, Zhang Y, Ottina KA, Lim D, et al. (2011). The mTOR-regulated phosphoproteome reveals a mechanism of mTORC1mediated inhibition of growth factor signaling. Science, 332:1317-1322.

[86] Yu Y, Yoon S, Poulogiannis G, Yang Q, Ma X, Villén J, et al. (2011). Phosphoproteomic analysis identifies Grb10 as an mTORC1 substrate that negatively regulates insulin signaling. Science, 332:1322-1326. 
[87] Yang L, Li P, Fu S, Calay E, Hotamisligil G (2010). Defective hepatic autophagy in obesity promotes ER stress and causes insulin resistance. Cell Metab., 11:467-478.

[88] Ozcan U, Cao Q, Yilmaz E, Lee A, Iwakoshi N, Ozdelen E, et al. (2004). Endoplasmic reticulum stress links obesity, insulin action, and type 2 diabetes. Science, 306:457-461.

[89] Liu X, Niu Y, Yuan H, Huang J, Fu L (2015). AMPK binds to Sestrins and mediates the effect of exercise to increase insulin-sensitivity through autophagy. Metab. Clin. Exp., 64:658-665.

[90] Jones RG, Thompson CB (2009). Tumor suppressors and cell metabolism: a recipe for cancer growth. Genes Dev, 23:537-548.

[91] Chen X, Wang Y, Tao J, Shi Y, Gai X, Huang F, et al. (2015). mTORC1 Up-Regulates GP73 to Promote Proliferation and Migration of Hepatocellular Carcinoma Cells and Growth of Xenograft Tumors in Mice. Gastroenterology, 149:741-752 e714.

[92] Witzig T, Reeder C, Han J, LaPlant B, Stenson M, Tun $\mathrm{H}$, et al. (2015). The mTORC1 inhibitor everolimus has antitumor activity in vitro and produces tumor responses in patients with relapsed T-cell lymphoma. Blood, 126:328-335.

[93] Faller WJ, Jackson TJ, Knight JR, Ridgway RA, Jamieson T, Karim SA, et al. (2015). mTORC1mediated translational elongation limits intestinal tumour initiation and growth. Nature, 517:497-500.

[94] Wei JL, Fu ZX, Fang M, Guo JB, Zhao QN, Lu WD, et al. (2015). Decreased expression of sestrin 2 predicts unfavorable outcome in colorectal cancer. Oncol Rep, 33:1349-1357.

[95] Maiuri MC, Malik SA, Morselli E, Kepp O, Criollo A, Mouchel PL, et al. (2009). Stimulation of autophagy by the p53 target gene Sestrin2. Cell Cycle, 8:15711576.

[96] Zhao B, Shah P, Budanov AV, Qiang L, Ming M, Aplin A, et al. (2014). Sestrin2 protein positively regulates AKT enzyme signaling and survival in human squamous cell carcinoma and melanoma cells. J Biol Chem, 289:35806-35814.

[97] Chen YR, Zweier JL (2014). Cardiac mitochondria and reactive oxygen species generation. Circ Res, 114:524-537.

[98] Ma X, Liu H, Foyil S, Godar R, Weinheimer C, Diwan A (2012). Autophagy is impaired in cardiac ischemiareperfusion injury. Autophagy, 8:1394-1396.

[99] Esposti D, Domart M, Sebagh M, Harper F, Pierron G, Brenner C, et al. (2010). Autophagy is induced by ischemic preconditioning in human livers formerly treated by chemotherapy to limit necrosis. Autophagy, 6:172-174.

[100] Sheng R, Zhang L, Han R, Liu X, Gao B, Qin Z (2010). Autophagy activation is associated with neuroprotection in a rat model of focal cerebral ischemic preconditioning. Autophagy, 6:482-494.

[101] Yan L, Sadoshima J, Vatner DE, Vatner SF (2009). Autophagy in ischemic preconditioning and hibernating myocardium. Autophagy, 5:709-712.

[102] Gottlieb RA, Mentzer RM (2010). Autophagy during cardiac stress: joys and frustrations of autophagy. Annu Rev Physiol, 72:45-59.

[103] Mariani J, Ou R, Bailey M, Rowland M, Nagley P, Rosenfeldt F, et al. (2000). Tolerance to ischemia and hypoxia is reduced in aged human myocardium. J. Thorac. Cardiovasc. Surg., 120:660-667.

[104] Reznick RM, Zong H, Li J, Morino K, Moore IK, Yu $\mathrm{HJ}$, et al. (2007). Aging-associated reductions in AMP-activated protein kinase activity and mitochondrial biogenesis. Cell Metab, 5:151-156.

[105] Quan N, Sun W, Wang L, Chen X, Bogan JS, Zhou X, et al. (2017). Sestrin2 prevents age-related intolerance to ischemia and reperfusion injury by modulating substrate metabolism. FASEB J, 31:4153-4167.

[106] Quan N, Wang L, Chen X, Luckett C, Cates C, Rousselle T, et al. (2018). Sestrin2 prevents agerelated intolerance to post myocardial infarction via AMPK/PGC-1 $\alpha$ pathway. J. Mol. Cell. Cardiol., $115: 170-178$ 